\title{
Shape Correction of Thin Mirrors
}

\author{
Keith Patterson* and Sergio Pellegrino ${ }^{\dagger}$ \\ California Institute of Technology, Pasadena, CA 91125
}

\begin{abstract}
Future large space observatories will require large apertures to provide better resolution and greater light gathering power; thin mirror technologies provide one possible route for addressing this need. This paper presents a study of a $10 \mathrm{~m}$ diameter sparse aperture based on a collection of thin, active mirror segments with identical initial shapes. A preliminary design for a $1 \mathrm{~m}$ diameter mirror segment is proposed and an investigation into the performance of this design is carried out utilizing finite element modeling tools. The results indicate that it is possible to adapt the generic segment shapes to fit the local mirror shape, and achieve near diffraction-limited performance through the use of lightweight, surface-parallel actuators. These actuators may also be used for thermal compensation. Additionally, a design for a scaled $10 \mathrm{~cm}$ diameter prototype mirror to test and validate the envisioned scheme is presented.
\end{abstract}

\section{Introduction and Background}

Space-based telescopes have been constrained by the size of available launch vehicles but this limitation will finally be removed with the launch of the first folding primary mirror in the James Webb Space Telescope, currently under construction. This is a major step forward in space telescope technology, however the cost and complexity associated with folding mirror architectures and the size limitations still inherent in this approach will ultimately necessitate even more radical approaches. Considering the time it takes for a new space observatory concept to reach maturity, the search for such alternatives should begin now.

We have recently participated in a study of large space apertures sponsored by the Keck Institute of Space Studies (KISS). ${ }^{1}$ This study put forward the concept of forming a large mosaic mirror through onorbit self-assembly of relatively small mirror segments mounted on separate low-cost spacecraft, building on recent developments in autonomous self-assembly in space. ${ }^{2-5}$ The spacecraft would dock and become mechanically connected together and the mirrors would adjust their shape to form a single coherent mirror surface. As a follow-on to this study, we have been studying the feasibility of a low-cost technology for lightweight mirrors to be used on each of these spacecraft.

The thin mirrors that we are considering would have a thickness on the order of a millimeter and diameter on the order of $1 \mathrm{~m}$. Active shape correction would be used to achieve and retain the required shape accuracy.

Lightweight mirrors known as Actuated Hybrid Mirrors ${ }^{6}$ are shown in Figure 1. These mirrors utilize a precision optical facesheet supported by a stiff, lightweighted backing structure with actuators attached to the rear. In order to further reduce the mirror mass, we propose to do away with the backing structure and attach the actuators directly to the back of the facesheet. The actuators would provide a layer of surface parallel actuation that provides internally reacted forces to provide the necessary shape adjustments. In future, this approach could conceivably be extended to thin mirrors that can be rolled up and stowed for launch, along the lines suggested by Romeo et al. ${ }^{7}$

Doubly-curved thin films mirrors have been studied extensively. Mirror substrates were formed by casting polyimide or other polymers on high-quality mandrels and then depositing a reflective coating of aluminum on one side of the substrate. A variety of different structural configurations have been considered, including pressurized inflatables, edge-tensioned flat surfaces, or freestanding shell structures. Boundary actuation provides of thin shell mirrors has been studied. ${ }^{8}$ A review of processes aimed at minimizing surface roughness, thickness variation, and thermal expansion has been compiled by de Blonk et al. ${ }^{9}$

\footnotetext{
*Graduate Student, Graduate Aerospace Laboratories, 1200 E. California Blvd. MC 205-45. kdpatter@caltech.edu

$\dagger$ Joyce and Kent Kresa Professor of Aeronautics and Professor of Civil Engineering, Graduate Aerospace Laboratories, 1200 E. California Blvd. MC 301-46. AIAA Fellow. sergiop@caltech.edu
} 


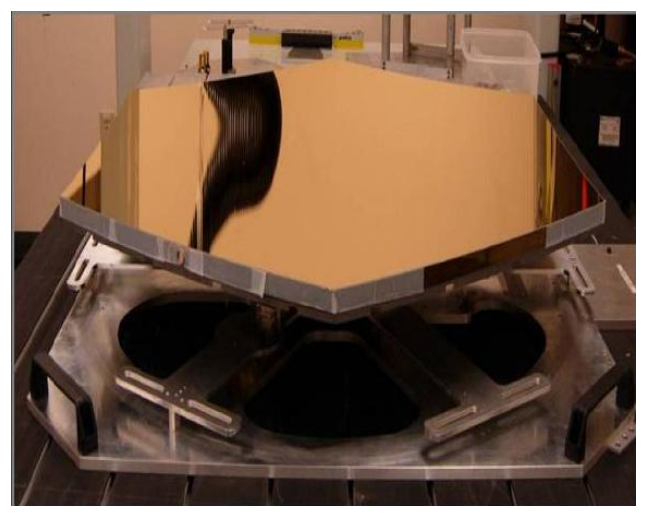

Figure 1. Actuated Hybrid Mirror with silicon carbide backing structure, embedded ceramic actuators, and thin facesheet. ${ }^{6}$

Surface parallel actuation schemes for thin mirrors have also been studied extensively. An early demonstration of a thin deformable mirror using piezoelectric elements that subdivide the mirror surface into large triangles were bonded to the backside of a $0.5 \mathrm{~m}$ mirror was shown by Kuo. ${ }^{10}$ There have been numerous other demonstrations of surface parallel actuation on thin mirror substrates, as described next. The design in Figure 2 involves a flat silicon substrate with a layer of deposited PZT patches. ${ }^{11}$ By varying the voltage applied to the different patches, various Zernike mode deformations were demonstrated. PZT has good actuation characteristics but is brittle and hence great care is required to ensure mechanical integrity of the actuating patches.

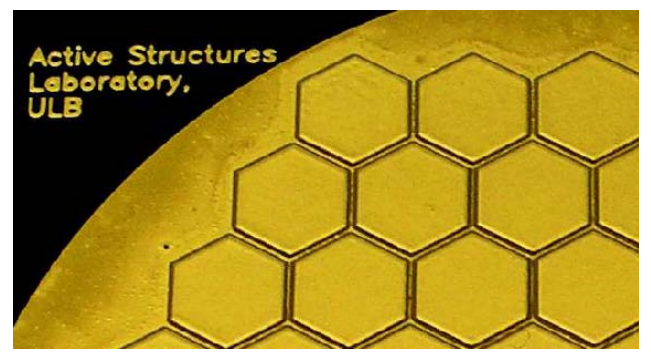

Figure 2. Silicon bimorph mirror with hexagonal PZT patches. ${ }^{11}$

Another possible actuator material is the piezoelectric polymer PVDF. Chen et al. ${ }^{12}$ built a thin mirror, shown in Figure 3, to demonstrate the use of PVDF active elements instead of traditional ceramic actuators, such as PZT or PMN. Sobers et al. ${ }^{13}$ constructed a tensioned, PVDF membrane mirror with the electrode pattern shown in Figure 4. Pearson et al. ${ }^{14}$ constructed a polyimide membrane mirror attached to a stiff edge structure and with a continuous layer of PVDF cast on the backside of the membrane. Figure 5 shows this mirror, first in its initial, slack configuration and then taut after actuating the PVDF layer. Xinetics recently filed a patent for a system entailing a membrane with a transducer system mounted onto it. ${ }^{15}$

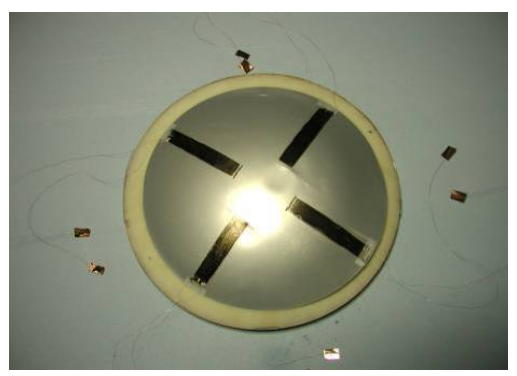

Figure 3. Mirror with premade discrete PVDF actuators bonded onto the backside. ${ }^{12}$ 


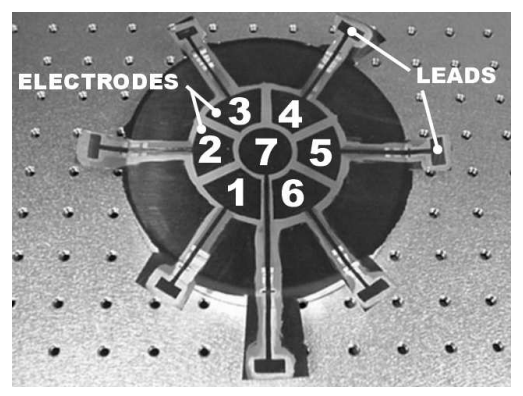

Figure 4. PVDF mirror with 7 active regions. ${ }^{13}$

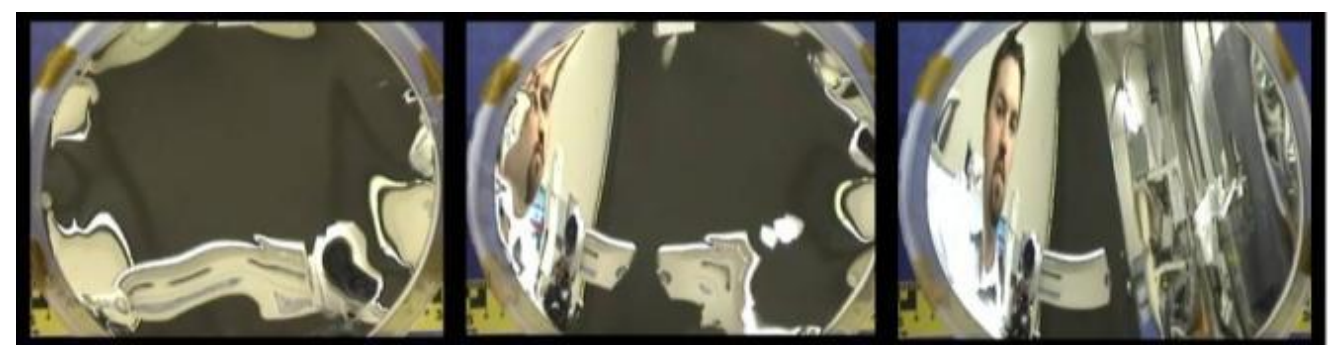

Figure 5. Thin mirror bonded to unpatterned PVDF actuating layer. ${ }^{14}$

A key advantage of the proposed approach is that, unlike existing primary mirror architectures where the stiffness of the mirror structure is such that the shape of the mirror can be changed only by small amounts, in the present scheme the use of a flexible structure and extensive actuation provides a much wider range of shape adaptability. This property opens up new possibilities, for example initially identical mirror segments can be used in various off-axis positions and the segments can also be moved around to reconfigure the shape of the aperture, and thus modify the point spread function (PSF) of the telescope. ${ }^{16}$

This paper presents a review of a methodology for analyzing mirror shape correction, followed by the conceptual design of a $1.0 \mathrm{~m}$ diameter mirror segment suitable for a $10 \mathrm{~m}$ mosaic aperture. The paper is laid out as follows. Section II reviews our technique for determining the best shape correction possible for any chosen mirror and actuating elements. Section III presents a $10 \mathrm{~m}$ diameter mirror architecture based on $1 \mathrm{~m}$ diameter active segments, whose design is presented in the following section. Next, Sections V and VI present a finite element study of the active segment and its predicted performance. Section VII report on ongoing efforts to construct a 1:10 scale model prototype of this segment, and Section VIII concludes the paper.

\section{Shape Correction}

The root-mean-square (RMS) surface error is a simple scalar measure of shape-related performance. It is particularly convenient for comparison purposes and it will be utilized as a criterion for mirror design. ${ }^{17}$ It should be noted that in a real mirror there are several different factors contributing to the RMS error. The high spatial frequency component of the RMS error will be governed by the mirror surface roughness; this is an issue related to manufacturing techniques and processes that bears little relation with shape correction. On the other hand, minimization of the low- to mid-frequency components of the RMS error may be achieved through the use of a sufficient number of actuators to bend or stretch the mirror into the desired shape.

The numerical techniques for mirror surface control are well established and will be reviewed here. Consider a general mirror surface with $m$ sampling points or nodes distributed on the surface, and an associated control system with $n$ actuators. Associated with the $i^{\text {th }}$ actuator is a column vector, $\mathbf{a}_{i} \in$ $\Re^{m}, i=1 . . n$, formed by finding the nodal deflections of a finite element model of the structure due to a unit input (e.g. 1 volt) to the associated actuator, and turning off all other actuators. This column vector is known as the influence vector, since it determines the influence that the actuator has on the mirror surface. It is linearly independent from the other vectors, corresponding to the other actuators. Example influence 


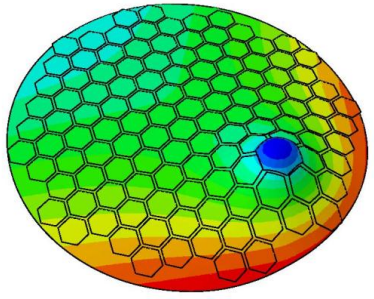

(a)

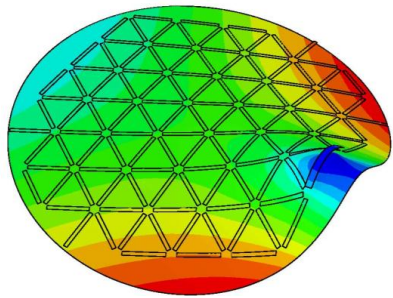

(b)

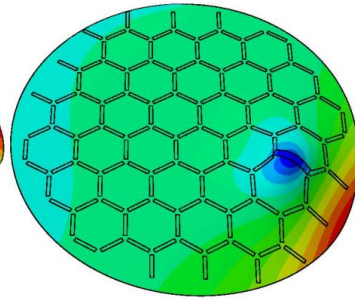

(c)

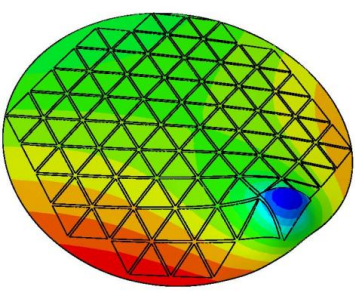

(d)

Figure 6. Some example influence functions.

functions are shown in Figure 6. The influence vectors are assembled into the influence matrix, $\mathbf{A}$ :

$$
\mathbf{A}=\left[\begin{array}{llll}
\mathbf{a}_{1} & \mathbf{a}_{2} & \ldots & \mathbf{a}_{n}
\end{array}\right] \in \Re^{m \times n}
$$

The assumption is made that all deviations from the initial surface shape are small. This is easily justified by noting that most surface shape adjustments are on the order of visible wavelengths (hundreds of nanometers), whereas the characteristic mirror dimensions are on the order of centimeters or meters. This assumption allows linear combinations of the influence vectors to be used to predict the mirror deflections. Hence, the influence matrix can be used to transform a control vector, $\mathbf{u} \in \Re^{n}$, consisting of the actuator input values, into a shape deflection vector, $\boldsymbol{\delta} \in \Re^{m}$, which contains the deflection of all nodal points of the mirror mesh. Thus, the control vector and shape deflection vector are related via the influence matrix by:

$$
\mathrm{Au}=\boldsymbol{\delta}
$$

In general, the mirror will start in a given shape, $\mathbf{s}_{1} \in \Re^{m}$, different from the desired shape $\mathbf{s}_{2} \in \Re^{m}$. Then, the desired deflection vector is $\boldsymbol{\delta}=\mathbf{s}_{2}-\mathbf{s}_{1}$. Even assuming that the appropriate vector norm (e.g. 2-norm or RMS) of $\boldsymbol{\delta}$ is small, this deflection vector will, in general, not belong to the rangespace of $\mathbf{A}$. Therefore, the appropriate control vector would come from a least squares (LS) solution of Eq. (2).

For a general mirror mesh, it is desirable to weight each of the nodal deflections by an appropriate associated surface area, $S_{i}$, such that the fitting solution is made independent of the particular mesh structure. In this study, an approximation for these areas is found by projecting the mirror surface nodes down onto a plane, computing the Voronoi area ${ }^{18}$ for each node, and then projecting the areas back onto the mirror surface via the direction cosine of the surface normal. These area weights are arranged along the diagonal of the matrix, $\mathbf{W} \in \Re^{m \times m}$. Equation (2) is then modified as follows:

$$
\mathrm{WAu}=\mathbf{W} \boldsymbol{\delta}
$$

One possible approach to find the weighted LS solution is through reduced QR factorization of the matrix product WA. Briefly:

$$
\begin{gathered}
\mathbf{W A u}=\widehat{\mathbf{Q}} \widehat{\mathbf{R}} \mathbf{u}=\mathbf{W} \boldsymbol{\delta} \\
\widehat{\mathbf{R}} \mathbf{u}=\widehat{\mathbf{Q}}^{\mathrm{T}} \mathbf{W} \boldsymbol{\delta}
\end{gathered}
$$

Once the right hand side of (5) has been computed, then due to the fact that $\widehat{\mathbf{R}}$ is upper triangular, a simple back-substitution method is employed to compute $\mathbf{u}$. Then, re-application of $\mathbf{A}$ results in an approximation to the original $\boldsymbol{\delta}$. The difference between the approximation and the original is the residual vector or residual shape error, $\mathbf{r}=\mathbf{A u}-\boldsymbol{\delta} \in \Re^{m}$. Accounting for the weights in the residual, $\hat{\mathbf{r}}=\mathbf{W A u}-\mathbf{W} \boldsymbol{\delta} \in \Re^{m}$. For convenience, the weights in $\mathbf{W}$ are now changed to be the square roots of $S_{i}$ non-dimensionalized by the total mirror surface area. Thus, the 2-norm of $\hat{\mathbf{r}}$ is now equivalent to the RMS surface error with units of length:

$$
\|\hat{\mathbf{r}}\|_{2}=\sqrt{\hat{\mathbf{r}}^{\mathrm{T}} \hat{\mathbf{r}}}=\sqrt{(\mathbf{A u}-\boldsymbol{\delta})^{\mathrm{T}} \mathbf{W}^{2}(\mathbf{A u}-\boldsymbol{\delta})} \equiv \sqrt{\frac{\sum_{i} r_{i}^{2} S_{i}}{\sum_{i} S_{i}}}
$$




\section{Large Aperture Study}

A sparse aperture with a diameter of approximately $10 \mathrm{~m}$ is shown in Figure 7; it is formed by 73 circular segments. To obtain a better fill factor, hexagonal segments would be more appropriate, however for this study circular segments were chosen for simplicity and for the convenience of being able to use Zernike polynomials (orthogonal functions over the unit disk) to describe the shape error of each segment.

The aperture is assumed to be part of a simple Cassegrain-style telescope with a parabolic primary mirror. The central segment may not be needed depending on the size of the hole required to pass light through the primary aperture into the focal plane detector, after reflection off of a secondary mirror. For simplicity, however, the central segment is included in the following analysis. The focal length of the paraboloid surface is chosen to be $10 \mathrm{~m}$, giving the mirror an $f$-number of $f / 1$.

The segments are assumed to be initially spherical with a radius to be determined and all identical. Making and testing 73 identical spherical segments would cost much less than the required number of off-axis parabolic segments. However, even with proper rigid body positioning of the segments within the aperture, there will be shape errors due to the difference between the chosen initial sphere and the desired local parabolic surface. The segments must have sufficient shape correction capability to reduce this shape error to a small fraction of the wavelength of light, in order to attain diffraction-limited telescope performance. The reference wavelength used throughout this paper is $633 \mathrm{~nm}$ (red light).

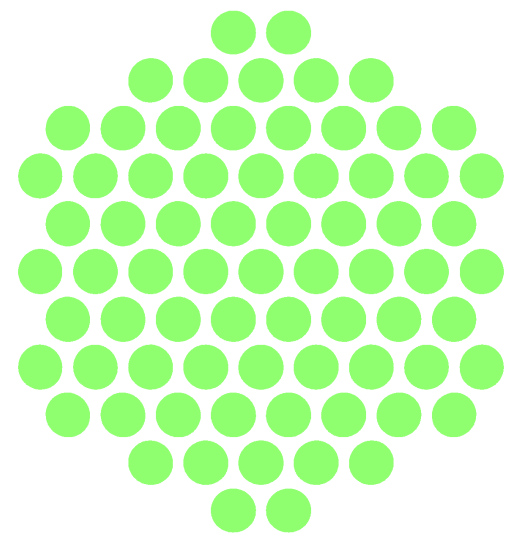

Figure 7. A $10 \mathrm{~m}$ sparse aperture containing $731.0 \mathrm{~m}$ diameter segments. The ideal parent surface of the mirrors is assumed to be parabolic with a focal length of $10 \mathrm{~m}$.

\section{Segment Design}

As mentioned previously, the segments are all initially identical. We consider a laminated structure consisting of several patterned layers, as follows.

- A $1 \mathrm{~mm}$ thick, passive, continuous layer with 42 radial tabs around the edge, Figure 9. This layer will be assumed to be made of Kapton with stiffness of 8.5 GPa.

- A $1 \mathrm{~mm}$ thick, active continuous layer with electrodes allowing each region to be actuated individually, Figure 8. The assumed material here is PVDF with a stiffness of $3 \mathrm{GPa}$ and piezoelectric coefficient $d_{31}=23 \times 10^{-12} \mathrm{~m} / \mathrm{V}$.

- A $1 \mathrm{~mm}$ thick, active triangular lattice consisting of strip actuators also made of PVDF. See Figure 9.

- A $10 \mathrm{~mm}$ thick ring also made of PVDF around the edge to provide boundary support in between the tabs, Figure 9.

A cartoon cross-section of the mirror segment is shown in Figure 10. The outer edge of the tabs of the substrate layers are supported by actuators perpendicular to the surface that can move up and down in order to modify the edge profile of the segment. 


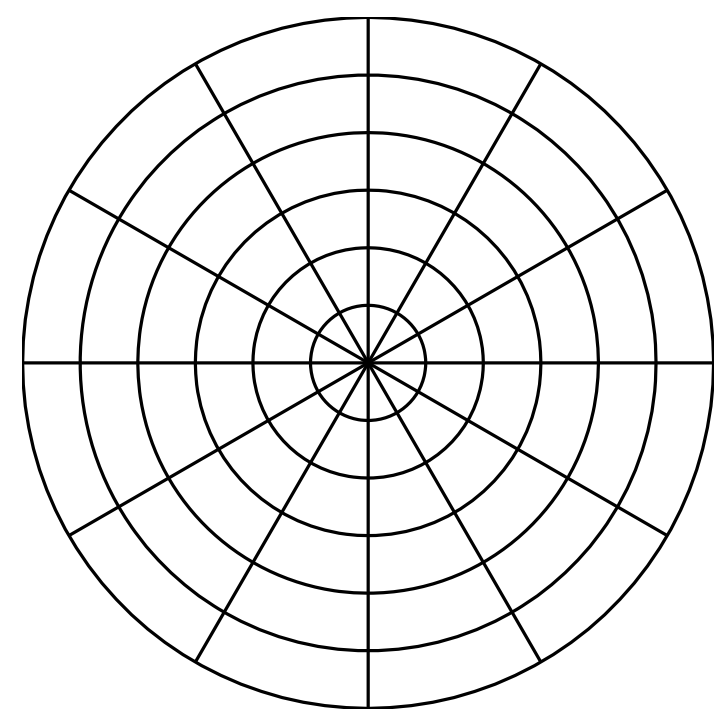

Figure 8. Layout of 72 actuating patches in the first active layer.

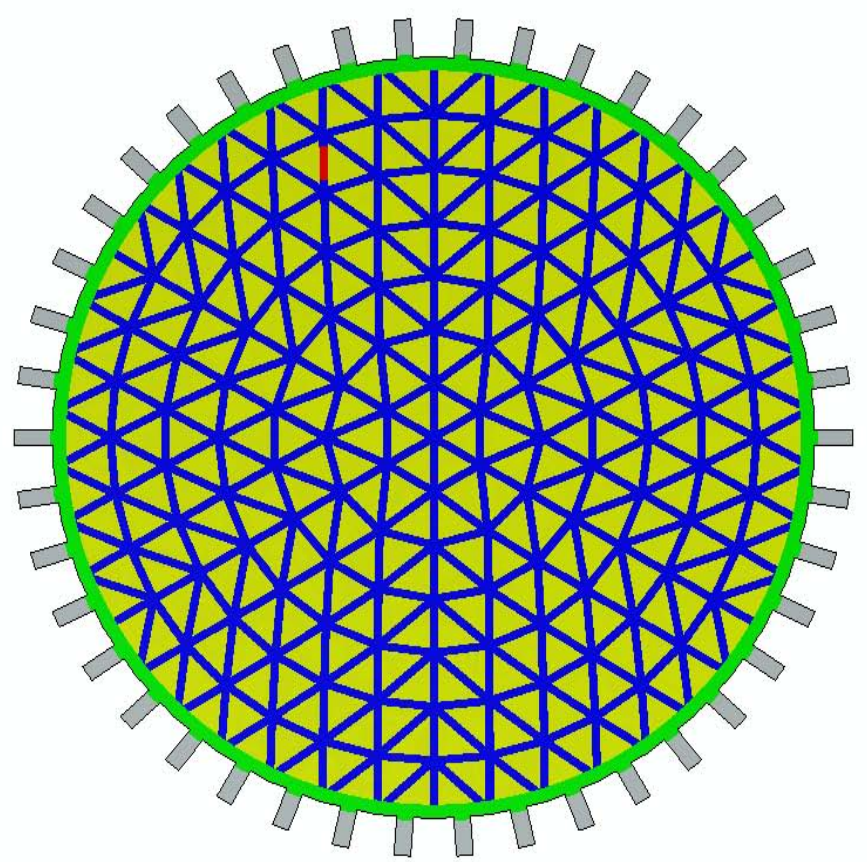

Figure 9. Layout of various active regions on mirror segment backside. Shown are the lattice of 462 strip actuators (blue), with a single strip being activated (red). Underneath this lattice layer is a continuous layer of PVDF broken into 72 sections laid out on a polar grid (yellow, divisions shown in Figure 8). The edge is thickened (green), while the ends of the nonreflective tabs (gray) would be clamped into a supporting ring or held by boundary actuators. 


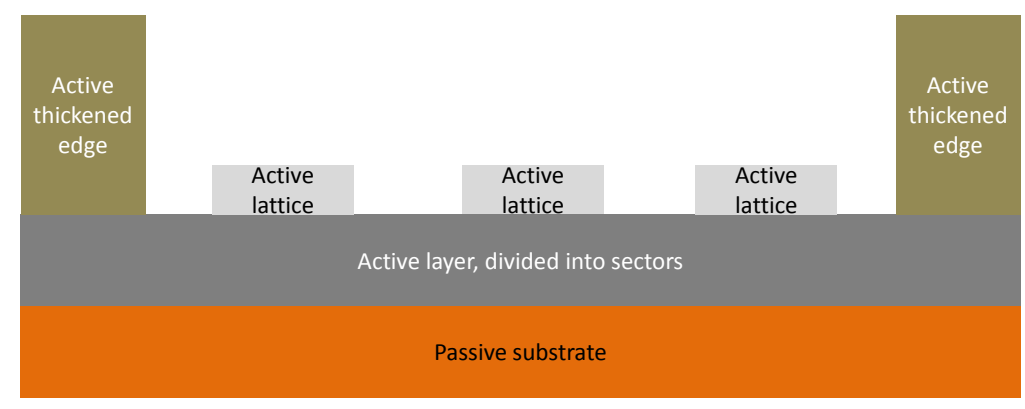

Figure 10. Schematic showing the various layers of the mirror structure. There is one passive layer, and three active layers, including: a single continuous layer, a lattice of strips, and a thickened edge to provide adjustable support in between clamped tabs.

\section{Finite Element Model}

A Finite Element Model (FEM) of the mirror and its actuators was created using the Abaqus/Standard 6.8-3 finite-element software and its Python automation scripting interface. The mirror was modeled as a single continuous surface divided into regions with uniform properties. Each region was defined as a laminated shell with the thickness and properties of each layer defined in Abaqus. The shell elements were fully integrated, finite membrane strain shell elements (S4T and S3T in Abaqus) and, due to the unavailability of piezoelectric shell elements in Abaqus, the expansion (and contraction) of the actuating layers was modeled by defining an appropriate coefficient of thermal expansion for each region and using temperature fields to provide the required actuation.

After the surface had been divided up and a fine mesh created, the influence functions for each actuator were calculated from the reference Abaqus model by changing the material expansion coefficients on or off for the piezoelectric actuators, or by changing the boundary conditions for the supporting actuators. The results of each analysis were imported into Matlab and a series of mirror performance calculations, system performance, and visualization post-processing were carried out.

\section{Performance Results}

This section analyzes key aspects of the performance of the $10 \mathrm{~m}$ diameter sparse aperture.

\section{A. Zernike Modes}

Figure 11 shows the generic performance of the $1 \mathrm{~m}$ active mirror segment, plotted in terms of Zernike mode components. Zernike modes are commonly used in optics work. ${ }^{19}$ Note how the mirror performance, plotted on the left, decreases and the required actuator voltages, plotted on the right, increase as the Zernike mode order increases. This dropoff may be alleviated to some extent by increasing the density of actuators.

\section{B. Thermal Correction}

It is important to consider the thermal correction capability of the mirror segment. One possible approach is to make the mirror thermally stable by using a material with low coefficient of thermal expansion (CTE) for the passive layer and then counteract the thermal strains in the active layer by imposing equal and opposite piezoelectric strains. However, there is concern about how much of the actuation range would be taken up by such thermal correction. An alternative approach is to allow the mirror to grow and shrink thermally, and to correct only the corresponding curvature change using the actuators. This second approach is proposed for the present design.

Here is provided a simple scaling argument to show the correction capability of the mirror segment in response to temperature changes. The assumption is made that all mirror layers are of a uniform CTE in order to neglect print-through effects, and to treat the mirror as effectively a single layer for thermal considerations. Assuming that the mirror starts as a spherical shell with radius of curvature, $R$, and diameter, 


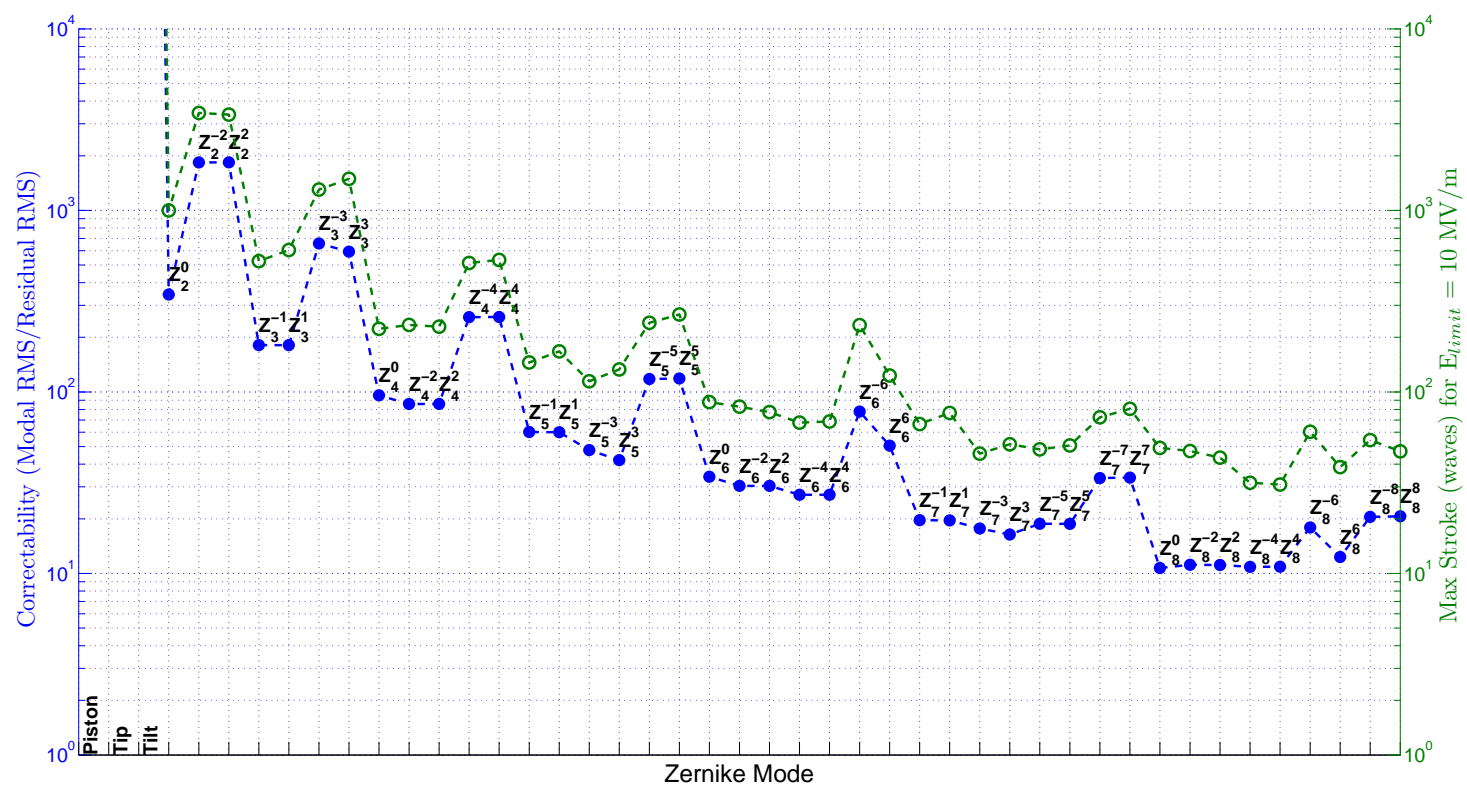

Figure 11. Generalized predicted performance of $1 \mathrm{~m}$ diameter circular segment with 462 strip, 72 annuli, and 42 edge support actuators, decomposed into Zernike modes.

$D$, with effective CTE, $\alpha$, then its curvature is:

$$
\kappa=\frac{1}{R}
$$

If the mirror undergoes a temperature change of $\Delta T$, then its change in curvature, assuming that the mirror is allowed to freely expand, is:

$$
\begin{gathered}
\Delta \kappa=\frac{1}{R(1+\alpha \Delta T)}-\frac{1}{R}=\frac{\alpha \Delta T}{R(1+\alpha \Delta T)} \\
\approx \frac{\alpha \Delta T}{R}
\end{gathered}
$$

This curvature change can be corrected by changing the focus mode of the mirror. Focus corresponds to the zernike mode, $Z_{2}^{0}$. The normalized mode is a parabolic function of the radial distance from the mirror center, $r$, equal to -1 in the mirror center, and increasing to 1 at the edge. It is scaled by the amplitude $a_{2}^{0}$. The additional curvature provided by this mode is the second derivative of the mode function:

$$
\begin{aligned}
& a_{2}^{0} Z_{2}^{0}=a_{2}^{0}\left(8\left(\frac{r}{D}\right)^{2}-1\right) \\
& \Delta \kappa_{z}=\frac{\partial^{2}}{\partial r^{2}}\left(a_{2}^{0} Z_{2}^{0}\right)=\frac{16 a_{2}^{0}}{D^{2}}
\end{aligned}
$$

Equating Eqn. 9 to Eqn. 11, and solving for the required modal amplitude:

$$
a_{2}^{0}=\frac{\alpha \Delta T D^{2}}{16 R}
$$

Note how the amplitude decreases if the mirror is flatter. In the limit, a flat mirror does not curve thermally and therefore would require no focus adjustment. For an effective material $\mathrm{CTE}$ of $10 \mathrm{ppm} /{ }^{\circ} \mathrm{C}$, a 
temperature change of $100{ }^{\circ} \mathrm{C}$, mirror diameter of $1.0 \mathrm{~m}$, and nominal radius of curvature $20 \mathrm{~m}$, the required focus mode amplitude is about $3 \mu \mathrm{m}$, or 5 waves. This amplitude should be handled easily in this design, since the maximum practical stroke for focus, shown in Figure 11, is about 300 waves.

\section{Optimum Initial Shape of Mirror Segments}

For the complete aperture, it is desired to determine the best starting shape (initial radius of curvature) for the segments. This was determined by finding the minimal RMS residual of the aperture after the segments had all been given a certain initial curvature, and after all the actuators had provided the best possible error minimizing correction, i.e. to achieve the smallest difference between the local parabolic surface and the starting sphere. The RMS residuals were combined over all segments and this value has been plotted in Figure 12 against the starting radius. As a reference, the radius of curvature of the paraboloid on its axis is equal to $20 \mathrm{~m}$ and it makes sense that the optimal radius is a bit larger than $20 \mathrm{~m}$ because the paraboloid curvature decreases with increasing distance from the axis, and there are many more segments towards the perimeter of the aperture than towards the center.

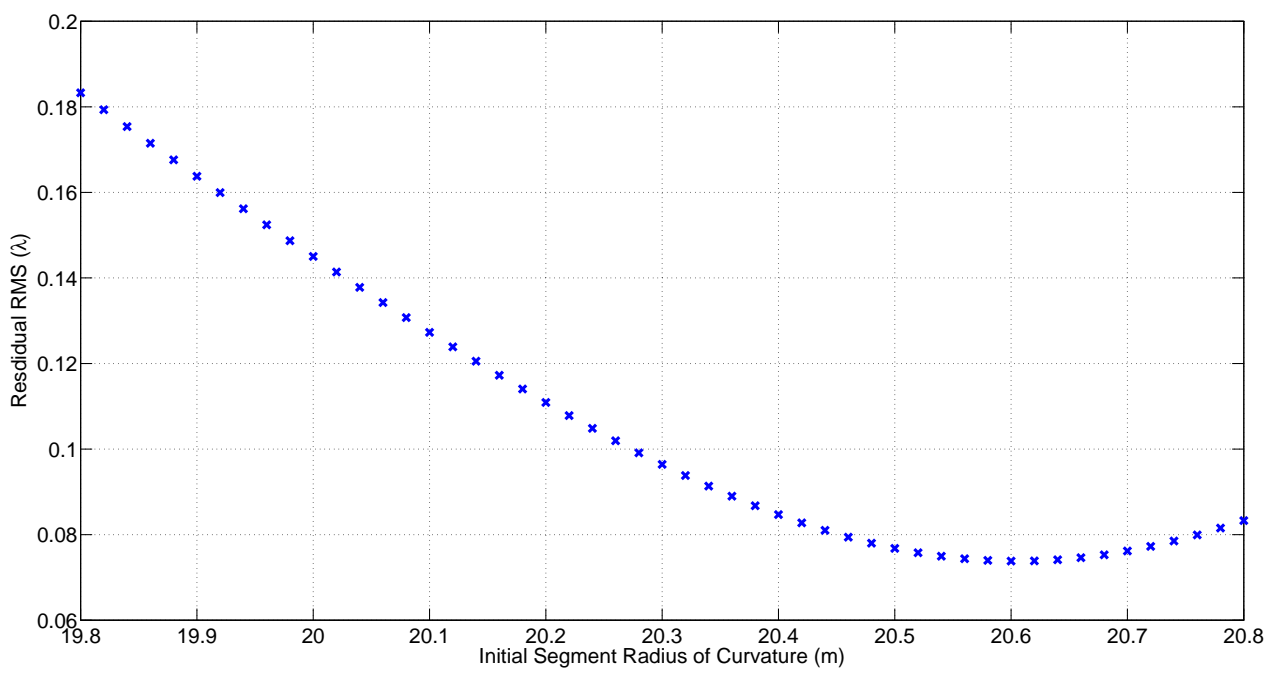

Figure 12. Residual RMS error for $10 \mathrm{~m}$ aperture formed by initially identical spherical segments, after correction by the embedded actuators. Scale is in units of $633 \mathrm{~nm}$ waves.

The optimal radius in this case turns out to be approximately 1.03 times the center radius. The mirror residual RMS is about 0.074 waves. With this optimal value in hand, it is possible to go back and investigate the segments' behavior at this design point. Figure 13 shows the initial shape errors at the optimal point. The segments in the middle rings have the least initial errors. Figure 14 displays the residual ripples that are left behind after the actuators have made their correction. This residual error has a length scale corresponding to the facesheet actuator spacing. For better performance, higher numbers of actuators would be needed to correct errors on smaller spatial frequency scales.

The required electric fields are shown in Figure 15. For the facesheet actuators, the required levels of electric fields could be achieved by using up to $500 \mathrm{~V}$ across $100 \mu \mathrm{m}$ thick sublayers of the PVDF material. Since the layers themselves are $1 \mathrm{~mm}$ thick in this design, electrodes would be required to be interdigitated at $100 \mu \mathrm{m}$ spacings to obtain the desired field levels without requiring too high voltages. The edge supporting actuators have to provide deflections up to about $300 \mu \mathrm{m}$ which could be achieved by piezoceramic-type actuators.

\section{Mirror Prototype}

All of the simulations provided so far were based upon linear models of material and mirror behavior. However, it is necessary to demonstrate a working prototype mirror in order to gain confidence in such a radical mirror design. A design for a 10:1 scale mirror with a diameter of $10 \mathrm{~cm}$ is proposed in this section. 


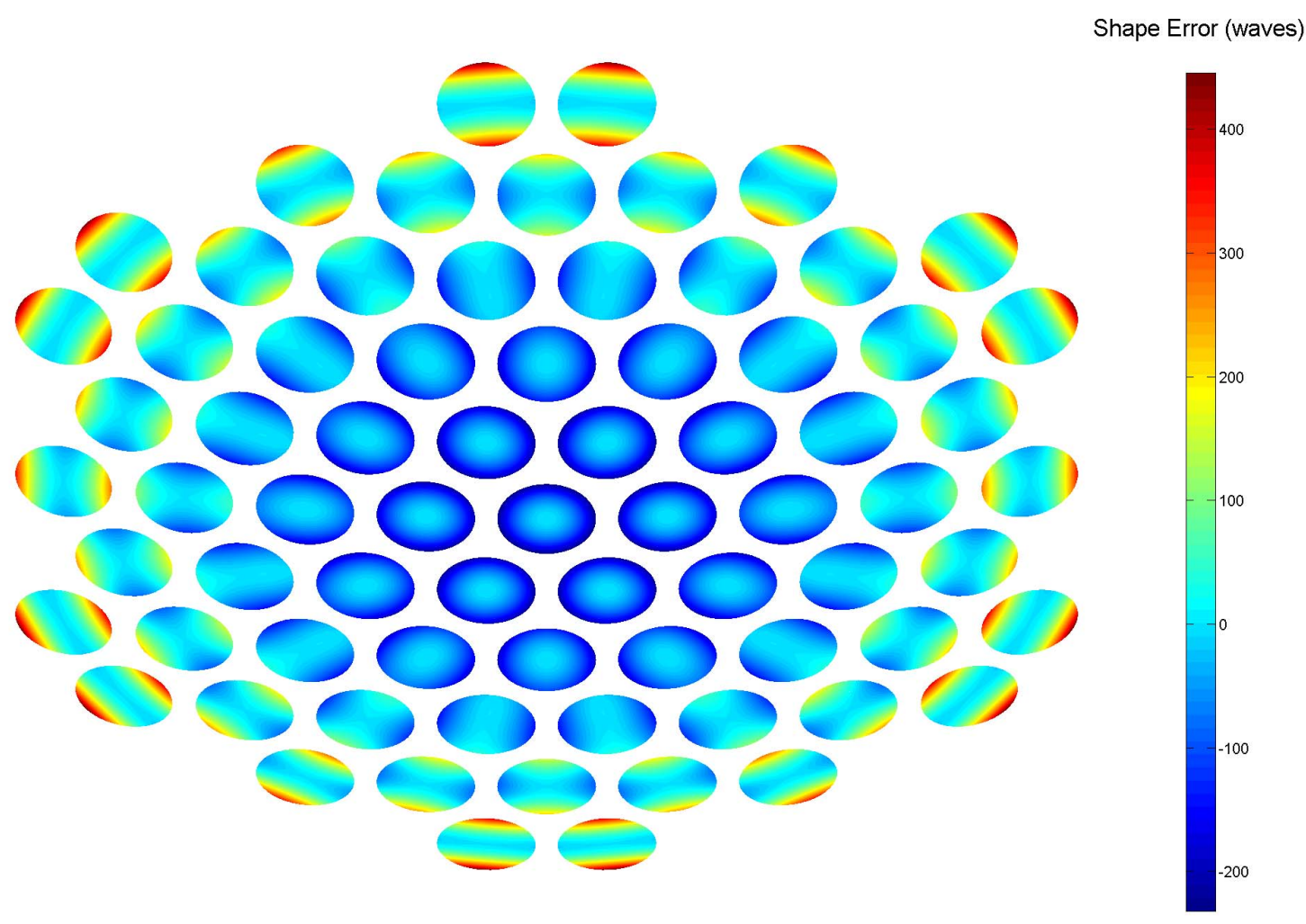

Figure 13. Initial shape errors of spherical segments forming a $10 \mathrm{~m}$ aperture. Scale is in units of $633 \mathrm{~nm}$ waves. 


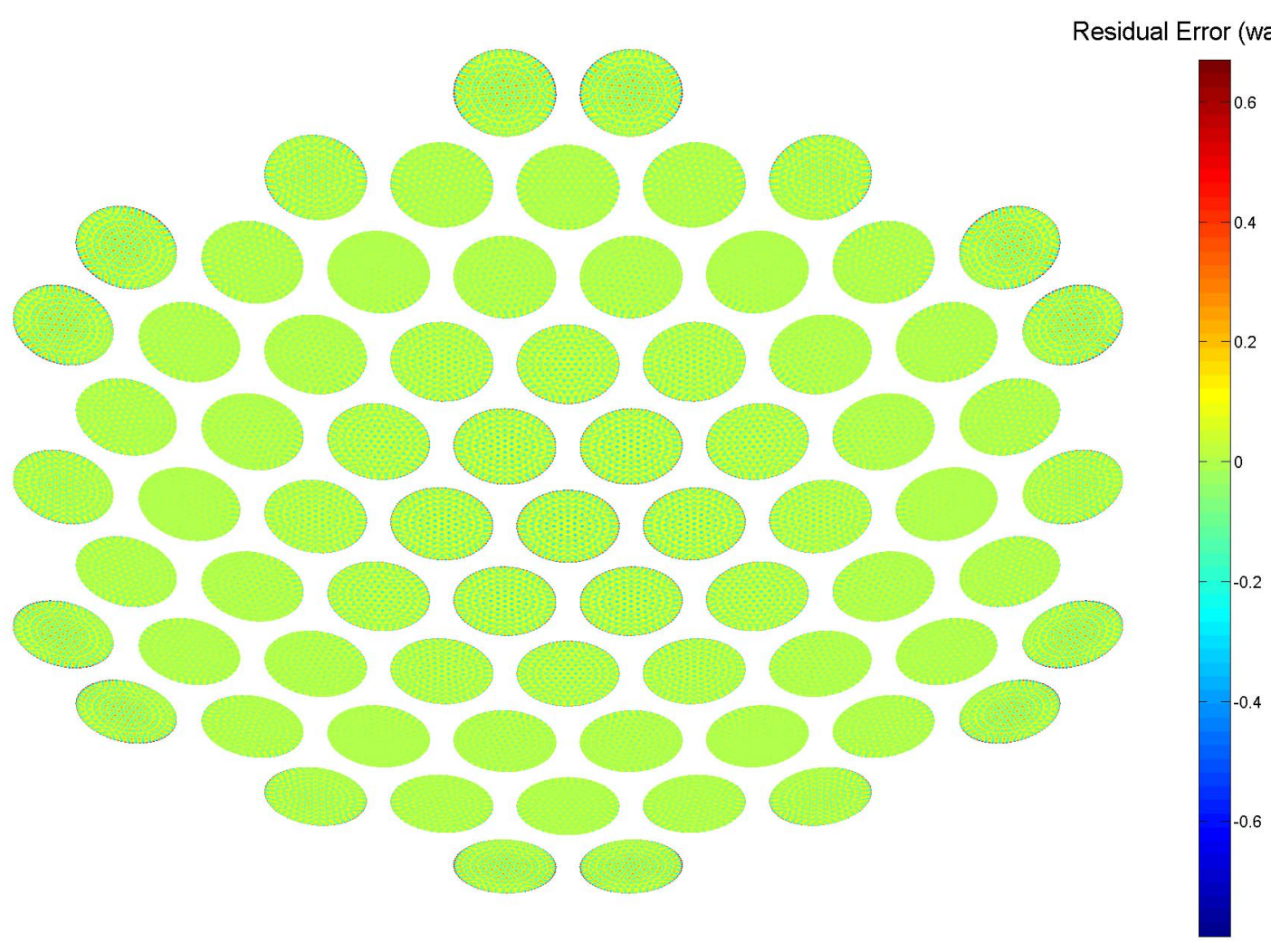

Figure 14. Residual errors of spherical segments in a $10 \mathrm{~m}$ aperture after correction. The initial curvature of the segments was the optimal value taken from Figure 12. Residual RMS is 0.074 waves.
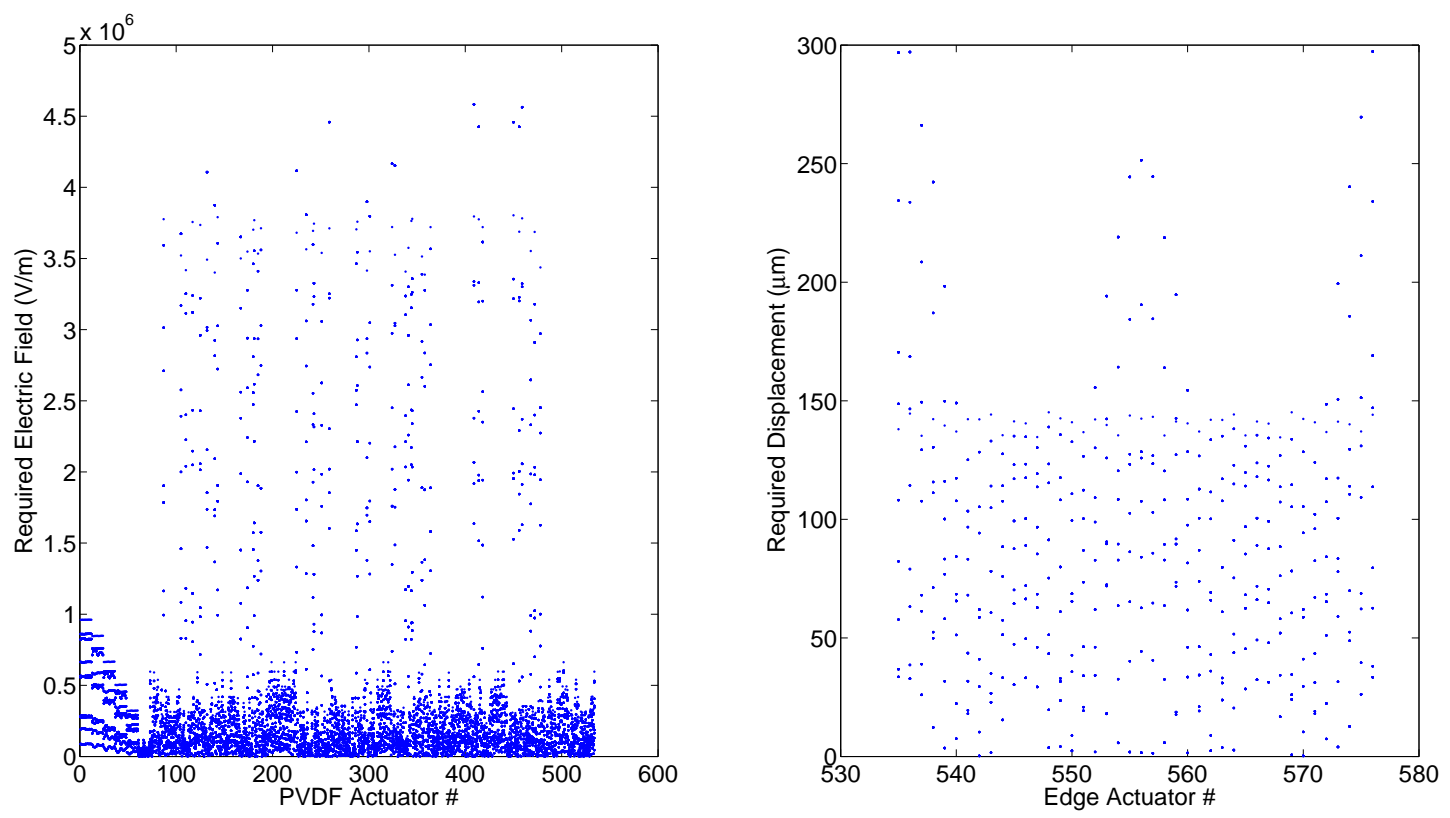

Figure 15. Required actuation values to achieve minimum RMS in Figure 14. 
Currently, efforts are underway to research and develop fabrication methodologies to manufacture such a prototype.

\section{A. Mirror Layers}

The design shown in Figure 16 incorporates a continuous active layer with its electrodes divided into 4 annuli. The patterned active layer above it consists of a lattice of 90 interconnected strip actuators. The electrodes for this upper layer are separated for individual addressing purposes. The top 3 layers essentially construct a "flex circuit" used to rout electrical lines from the mirror edge to the individual actuator electrodes. The thickest layers are the bottom passive layer and two active layers. These layers would be $100 \mu \mathrm{m}$ thick, while the other layers would be significantly thinner and should not affect the mirror mechanical behavior much. Not shown in this figure are intermediate bonding layers which are critical to prevent debonding during thermal processing or mirror operation. The layers would be built up from a polished mold and a reflective coating would be applied after separation of the structure from the mold.

\section{Cover $(25 \mu \mathrm{m})$ \\ Routing traces $(5 \mu \mathrm{m})$ \\ Via layer $(25 \mu \mathrm{m})$ \\ Electrodes $(5 \mu \mathrm{m})$ \\ Active layer $(100 \mu \mathrm{m})$ \\ Common ground $(5 \mu \mathrm{m})$ \\ Active layer $(100 \mu \mathrm{m})$ \\ Electrodes $(5 \mu \mathrm{m})$ \\ Passive layer $(100 \mu \mathrm{m})$}

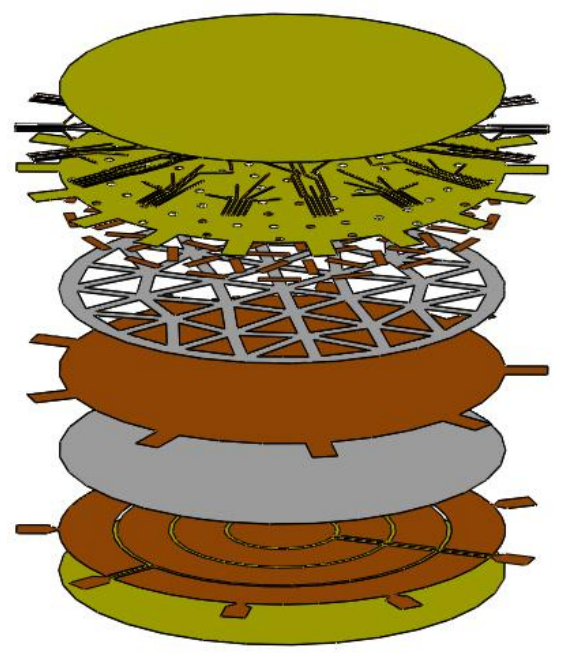

Figure 16. Exploded view of proposed $10 \mathrm{~cm}$ prototype mirror. The reflective surface, not shown in this view, is at the bottom of the passive layer.

\section{B. Mirror Support}

The edge of the mirror has 18 tabs which could be clamped into a ring as shown in Figure 17, but edge adjustments would then be prevented. To add edge adjustment capability, a cartoon cross-section of a modified ring integrated with piezoceramic actuators is shown in Figure 18. Pre-compressed piezoceramic stacks could provide submillimeter strokes. In order to position the mirror, the ring would be mounted onto a piston/tip/tilt stage, or a full 6 degree-of-freedom kinematic stage.

Another possibility is to directly link the mirror tabs to the spacecraft bus structure using individual actuators, without the need for a rigid ring. Moving all of the edge actuators synchronously would then provide limited rigid body adjustments without the need for a separate kinematic stage.

\section{Control Electronics}

Due to the design's large number of actuators, it becomes impractical to use a separate amplifier to provide the required large voltage to each actuator. Instead, it is proposed to charge up the individual actuators serially using a single amplifier board such as was demonstrated by Song in a similar application. ${ }^{20}$ This is possible because the PVDF material acts electrically like a small capacitor and, with its small leakage current, it is possible to periodically refresh its voltage on a short timescale. A schematic showing the components needed to provide feedback control of the mirror surface is shown in Figure 19. A wavefront sensor provides information about the mirror figure, a controller determines the appropriate actuator control 


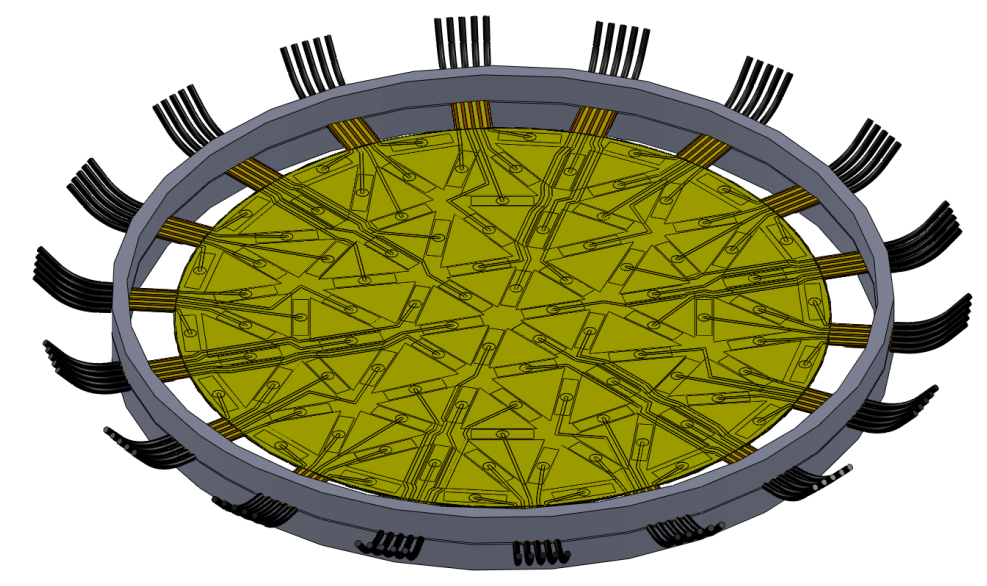

Figure 17. Simple option for mounting $10 \mathrm{~cm}$ prototype. This type of boundary condition would restrict mirror adjustments to axisymmetric deformations.

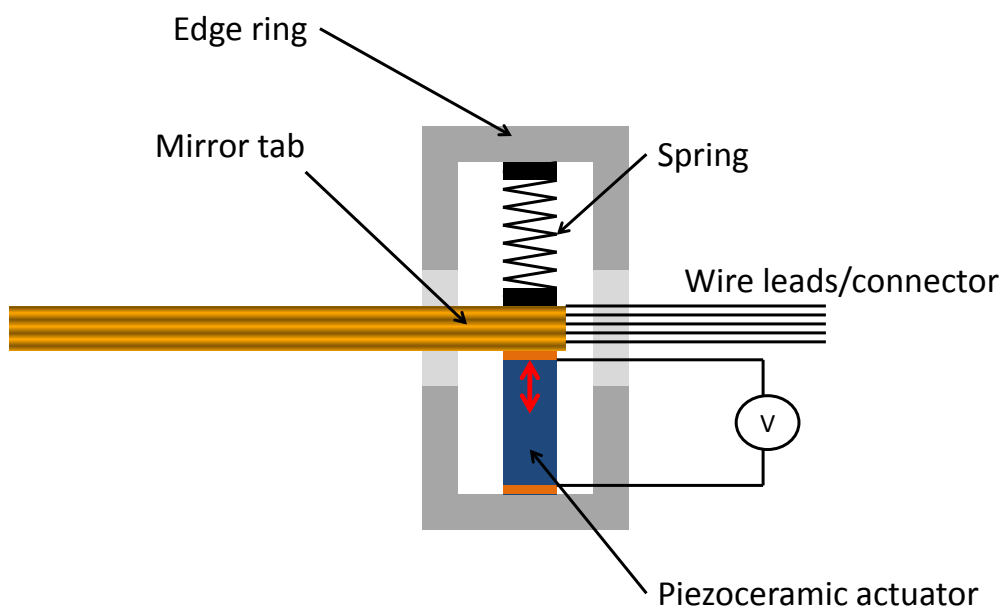

Figure 18. Example of showing how to incorporate piezoceramic actuators into the the supporting edge ring to provide edge actuation capability. 
voltages and, through the use of optical switches, cycles through each actuator and sets its voltage. This process continues through the mirror operation.

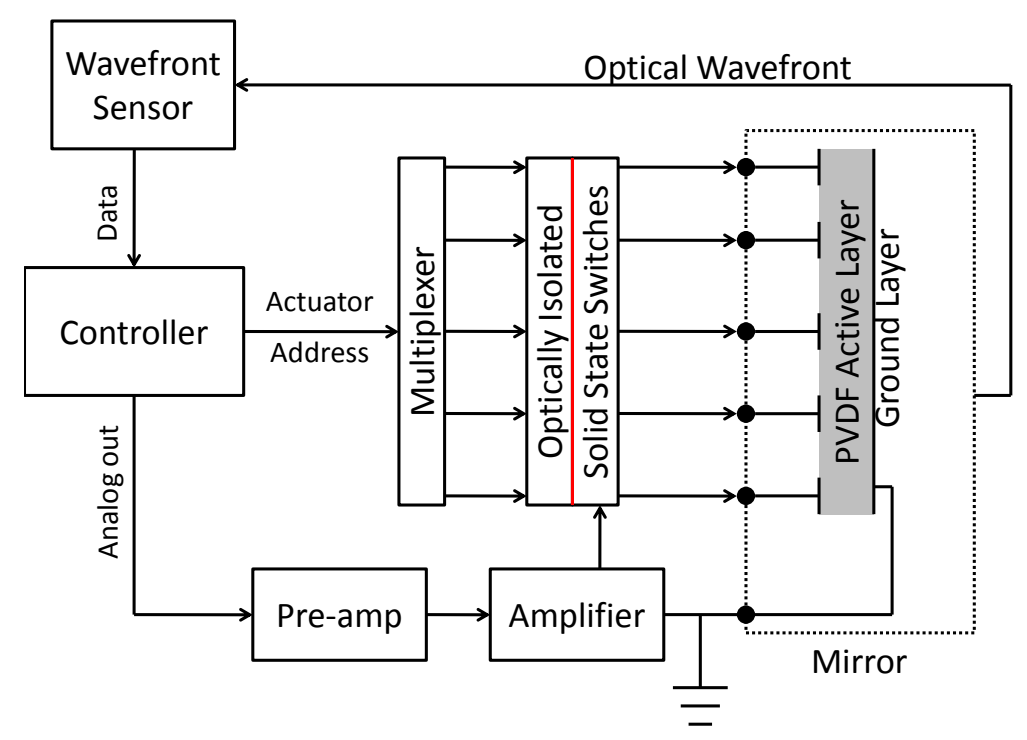

Figure 19. Schematic showing the various components needed to control the mirror surface figure.

\section{Predicted Performance}

The predicted generalized performance of this $10 \mathrm{~cm}$ prototype mirror design is shown in Figure 20. The maximum strokes of the scaled model are much lower than that of the full-scale model, however, it can be reasonably expected that the required strokes of a smaller mirror should also be smaller.

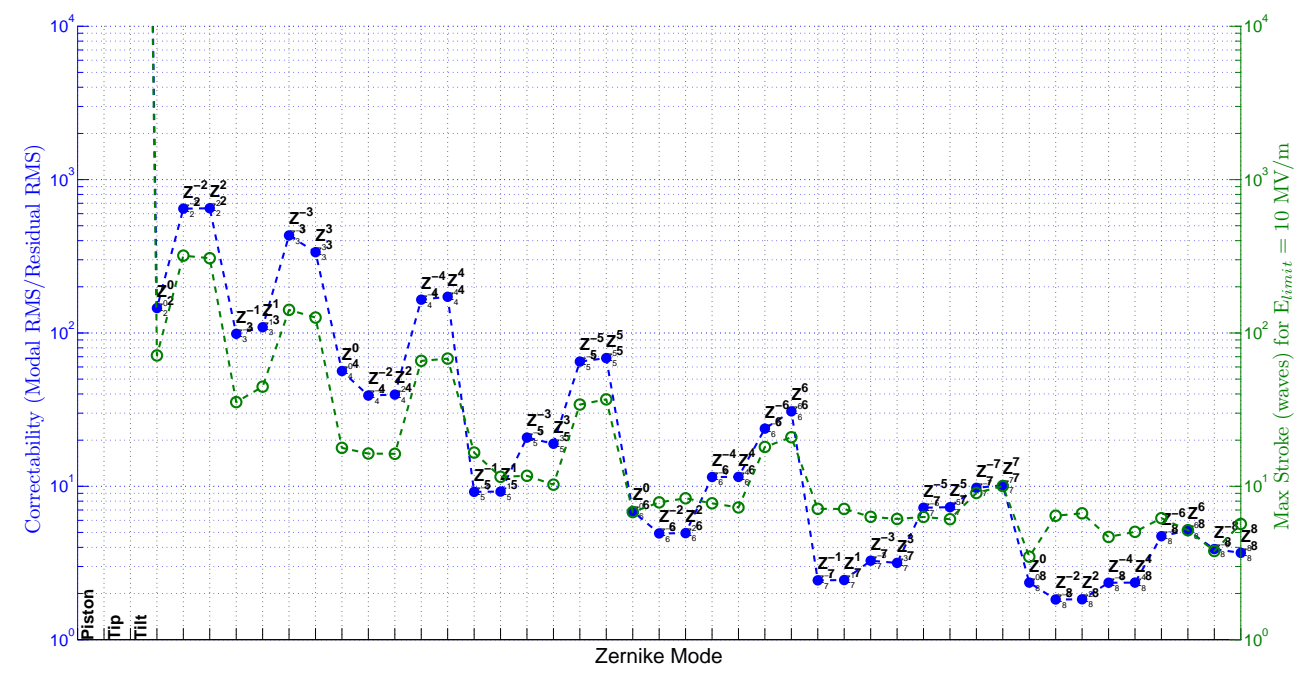

Figure 20. Predicted performance of $10 \mathrm{~cm}$ mirror, decomposed into Zernike modes. 


\section{Discussion and Conclusion}

It has been shown in this study that it may be feasible to construct a $10 \mathrm{~m}$ aperture out of identical, thin, low mass, $1 \mathrm{~m}$ diameter segments with active correction capability. Given a design incorporating hundreds of lightweight surface parallel actuators, as well as some supporting surface normal edge control actuators, it is possible to achieve residual shape errors smaller than a tenth of the wavelength of red light, and achieve diffraction-limited optical performance of the mirrors. By keeping the design of each segment identical, it is possible to lower the manufacturing costs of such a telescope, and allow for adaptability and expandability of the aperture.

A method to determine the initial segment design has also been demonstrated, and an argument given on how an aperture made of such active segments could accommodate large temperature swings during operation by self-correcting any curvature changes due to thermal expansion and contraction.

It is important to demonstrate the feasibility of this concept by creating a physical prototype. A conceptual design has been shown for a scaled $10 \mathrm{~cm}$ diameter mirror. It would consist of a laminated structure incorporating various passive and active layers, as well as electrical routing layers. The amount of control electronics required may be minimized by charging each actuator with a single amplifier in a serial manner, and cycling the voltages in order to keep the charges on the actuators refreshed. A few examples of mounting a prototype mirror have also been given.

Efforts currently involve the identification of fabrication schemes to construct the mirror, and selection of appropriate active, passive, and adhesive materials for use in its laminated stackup. Processes considered are for use in the making the smaller prototype, but there is an awareness of the need to allow for scalability to make larger mirror segments in the future. Further work is needed to resolve remaining fabrication issues, but if successful, such a mirror technology could promise to provide an technological improvement required for a large space aperture.

\section{Acknowledgments}

We thank Jim Breckinridge for helpful discussions of telescope design and Chiara Daraio, Eleftherios Gdoutos, and Andrew Shapiro for help and advice regarding the fabrication of a prototype. Financial support from the Keck Institute of Space Studies at Caltech is gratefully acknowledged.

\section{References}

\footnotetext{
${ }^{1}$ Breckinridge, J.B., Dooley, J., Ortiz, M. and Pellegrino, S., Large Space Apertures (LSA) Study Report, Keck Institute of Space Studies (2009).

${ }^{2}$ Katz, J.G. Estimation and Control of Flexible Space Structures for Autonomous On-Orbit Assembly, M.Sc. thesis in Aeronautics and Astronautics, MIT (2009).

${ }^{3}$ Natori, M.C., Ukegawa, K., "Concept of self-assembly of space structure systems using autonomous modules," 54th International Astronautical Congress of the International Astronautical Federation, 29 September - 3 October 2003, Bremen, Germany, IAC-03-U.1.01 (2003).

${ }^{4}$ Wokes, D., Smail, S., Palmer, P., Underwood, C., "Pose estimation for in-orbit self-assembly of the intelligent self-powered modules," AIAA Guidance, Navigation, and Control Conference 10 - 13 August 2009, Chicago IL, AIAA 2009-6291 (2009)

${ }^{5}$ Rodgers, L.P. Concepts and Technology Development for the Autonomous Assembly and Reconfiguration of Modular Space Systems, M.Sc. thesis in Mechanical Engineering, MIT (2005).

${ }^{6}$ Hickey, G., Barbee, T., Ealey M., and Redding, D. "Actuated hybrid mirrors for space telescopes," SPIE Astronomical Telescopes and Instrumentation 7731-71 (2010).

${ }^{7}$ Romeo, R.C., Meinel, A.B., Meinel, M.P. and Chen, P.C., "Ultra-lightweight and hyper-thin rollable primary mirror for space telescopes," in UV, Optical, and IR Space Telescopes and Instruments, Breckinridge, J.B., ed., vol. 4013. SPIE, pp. 634-639 (2000).

${ }^{8}$ Lindler, J. and Flint, E., "Robustness of thin film shells with discrete boundary actuation," 47th AIAA/ASME/ASCE/AHS/ASC Structures, Structural Dynamics, and Materials Conference (2006).

${ }^{9}$ de Blonk, B., Moore, J., Patrick, B., and Flint, E., "Membrane mirrors in space telescopes," in Recent Advances in Gossamer Spacecraft, Jenkins, C., ed., 45-108, American Institute of Aeronautics and Astronautics, Inc., Reston, Virginia (2006).

${ }^{10}$ Kuo, C.P., "A deformable mirror concept for adaptive optics in space," SPIE Vol. 1542 Active/Adaptive Optical Systems SPIE Vol. 1542 (1991).

${ }^{11}$ Rodrigues, G., Bastaits, R., Roose, S., Stockman, Y., Gebhardt, S., Schoenecker, A., Villon, P., and Preumont, A., "Modular bimorph mirrors for adaptive optics," in [Optical Engineering], Volume 48, Issue 3, pp. 034001-034001-7 (2009).

${ }^{12}$ Chen, Q., Natale, D., Neese, B., et al, "Piezoelectric polymers actuators for precise shape control of large scale space antennas," SPIE Smart Structures and Materials and Nondestructive Evaluation and Health Monitoring 07-0607 (2007).
} 
${ }^{13}$ Sobers, D.M. Jr., Agnes, G. S., Mollenhauer, D., "Smart structures for the control of optical surfaces," 44th AIAA/ASME/ASCE/AHS Structures, Structural Dynamics, and Materials Conference AIAA 2003-1559 (2003).

${ }^{14}$ Pearson, J., Moore, J., and Fang, H., "Large and high precision inflatable membrane reflector," 51st AIAA/ASME/ASCE/AHS/ASC Structures, Structural Dynamics, and Materials Conference, AIAA, Orlando, Florida (2010).

${ }^{15}$ Pearson, D.D., Cavaco, J.L., Roche, J., "Multichannel, surface parallel, zonal transducer system", U.S. Patent No. 7,683,524 B2. Washington, DC: U.S. Patent and Trademark Office (2010).

${ }^{16}$ Patterson, K., Pellegrino, S., and Breckinridge, J., "Shape correction of thin mirrors in a reconfigurable modular space telescope," SPIE Astronomical Telescopes and Instrumentation 7731-72 (2010).

${ }^{17}$ Bely, P., The Design and Construction of Large Optical Telescopes, Springer, New York (2003).

${ }^{18}$ Klein, R., Concrete and Abstract Voronoi Diagrams, Springer-Verlag, Berlin (1987).

${ }^{19}$ Born, M., Wolf, E., Principles of Optics, Pergamon Press, Oxford (1989).

${ }^{20}$ Song, H., Simonov, A., Vdovin, G., "Multiplexing Control of a Multichannel Piezoelectric Deformable Mirror," 5th International Workshop on Adaptive Optics for Industry and Medicine SPIE Vol. 6018 60181F-1 (2005). 